\title{
Effects of immunoglobulin plus prednisolone in reducing coronary artery lesions in patients with Kawasaki disease: study protocol for a phase III multicenter, open-label, blinded-endpoints randomized controlled trial
}

Si-Yuan Lin ${ }^{1 \dagger}$, Lan He ${ }^{1+}$, Li-Ping Xie', Yin Wang ${ }^{2}$, Yi-Xiang Lin ${ }^{1}$, Yin-Yin Cao ${ }^{1}$, Wei-Li Yan ${ }^{2 *}$, Fang Liu ${ }^{1 *}$ (D) and Guo-Ying Huang ${ }^{1 *}$

\begin{abstract}
Background: Kawasaki disease (KD) is an acute systemic vasculitis of unclear etiology that mainly affects infants and young children. Strategies to reduce the incidence and severity of coronary artery lesions (CALs), the determinant factor in the long-term prognosis of KD, are currently a focus of studies on KD. Corticosteroids, preferred in the treatment of the majority of vasculitides, are controversial in the treatment of acute KD. In this trial, we will evaluate whether the addition of prednisolone to standard intravenous immunoglobulin (IVIG) plus aspirin therapy can reduce the occurrence of CAL in Chinese patients with KD.

Methods: This is a multicenter, prospective, open-label, randomized controlled trial, which is expected to be conducted in more than 20 hospitals in China and aims to assess the efficacy and safety of IVIG + prednisolone treatment versus standard treatment. Patients with KD who fulfill the inclusion and exclusion criteria will be recruited and randomized (1:1) to receive either a large dose of IVIG (2 g/kg over 12-24 h with a maximum dose of $60 \mathrm{~g})+$ aspirin $30 \mathrm{mg} / \mathrm{kg} / \mathrm{d}$ or IVIG (2 g/kg over 12-24 h) + aspirin $30 \mathrm{mg} / \mathrm{kg} / \mathrm{d}$ + prednisolone (2 mg/kg/d with a maximum dose of $60 \mathrm{mg}$ tapered over 15 days after normalization of C-reactive protein concentration). The primary outcome will be the occurrence of CAL at 1 month of illness. The follow-up duration for each participant will be set as 1 year. Patients and treating physicians will be unmasked to group allocation.
\end{abstract}

\footnotetext{
* Correspondence: yanwl@fudan.edu.cn; liufang@fudan.edu.cn; gyhuang@shmu.edu.cn

${ }^{\dagger}$ Si-Yuan Lin and Lan He contributed equally to this work.

${ }^{2}$ Clinical Trial Unit, Children's Hospital of Fudan University, Shanghai 201102,

China

${ }^{1}$ Heart Center, Children's Hospital of Fudan University, Shanghai 201102,

China
}

(C) The Author(s). 2021 Open Access This article is licensed under a Creative Commons Attribution 4.0 International License, which permits use, sharing, adaptation, distribution and reproduction in any medium or format, as long as you give appropriate credit to the original author(s) and the source, provide a link to the Creative Commons licence, and indicate if changes were made. The images or other third party material in this article are included in the article's Creative Commons licence, unless indicated otherwise in a credit line to the material. If material is not included in the article's Creative Commons licence and your intended use is not permitted by statutory regulation or exceeds the permitted use, you will need to obtain permission directly from the copyright holder. To view a copy of this licence, visit http://creativecommons.org/licenses/by/4.0/. The Creative Commons Public Domain Dedication waiver (http://creativecommons.org/publicdomain/zero/1.0/) applies to the data made available in this article, unless otherwise stated in a credit line to the data. 
Discussion: This will be the first multicenter randomized controlled trial to evaluate the efficacy of IVIG + aspirin + prednisolone in Chinese pediatric patients with KD, which may provide high-level evidence for improving the initial treatment for acute KD.

Trial registration: ClinicalTrials.gov NCT04078568. Registered on 16 August 2018.

Keywords: Kawasaki disease, Primary treatment, Corticosteroid, Coronary artery lesions

\section{Background}

Kawasaki disease (KD) is an acute systemic vasculitis of unclear etiology that mainly affects children under 5 years of age [1]. KD has been reported worldwide and is more prevalent in Asia, especially in Japan, where the incidence rate was 330.2 and 309.0 per 100,000 children < 5 years of age in 2015 and 2016, respectively [2]. In Shanghai, China, the annual incidence rate of KD per 100,000 children $<5$ years is also increasing, ranging from 68.8 in 2013 to 104.6 in 2017 [3].

The development of coronary artery lesions (CALs) is the most important complication and the main determinant of the long-term prognosis of KD. CAL can contribute to coronary artery aneurysms, occlusion, myocardial ischemia, myocardial infarction, and even death, making it a major cause of acquired heart disease in developed countries [4]. Although large doses of intravenous immunoglobulin (IVIG) have been demonstrated to reduce the development of CALs [5, 6], the incidence of medium to giant coronary aneurysms remains as high as $3.4 \%$ in China [3]. Thus, focus has been placed on the means to provide aggressive treatment with superior efficacy in reducing the occurrence of CAL.

Corticosteroids, preferred in the treatment of the majority of vasculitides, are controversial in the acute treatment of KD. An early meta-analysis conducted by Wooditch et al. [7] indicated that using corticosteroids in aspirin-containing regimens for primary treatment of KD could reduce the incidence of coronary aneurysms, which was maintained when IVIG was added to the treatment regimen. In 2007, a randomized trial assessing the efficacy of the addition of a single pulsed dose of intravenous methylprednisolone to conventional IVIG therapy did not provide support for this conclusion [8]. Findings from another randomized trial in 2012 evaluating the efficacy of immunoglobulin plus prednisolone for preventing CAL showed benefits for children with severe KD in Japan based on the Kobayashi scoring system [9]. Similarly, a meta-analysis conducted by Zhu et al. revealed that corticosteroids plus conventional treatment may reduce the incidence of CAL in patients with $\mathrm{KD}$, and Chen et al. further demonstrated the importance of timing in another meta-analysis [10,11]. All results suggest that the therapy time, regimen, and target group require careful consideration in the use of corticosteroids for KD treatment. As a vasculitis, using corticosteroids in the early therapy of KD may benefit not only those who are at high risk of IVIG resistance but also all patients with KD.

In light of these findings, we have designed this trial, a phase III, multicenter, prospective, open-label, randomized controlled trial aimed at assessing the efficacy of the addition of prednisolone to conventional initial treatment in children with acute KD. This study will be conducted in more than 20 hospitals within the Chinese Kawasaki Disease Collaboration Network.

\section{Methods \\ Objectives}

1. To determine whether IVIG + aspirin + prednisolone combination therapy as the primary treatment is superior to conventional IVIG + aspirin treatment in reducing the occurrence of CAL in patients with KD.

2. To optimize the initial treatment regimen for acute KD.

\section{Trial design}

This is a multicenter, prospective, open-label, randomized controlled trial that will be carried out in more than 20 hospitals with adequate experience in diagnosing and treating $\mathrm{KD}$. With written informed consent, patients who meet the eligibility criteria will be enrolled and randomly assigned in a $1: 1$ ratio to the control (receiving $2 \mathrm{~g} / \mathrm{kg}$ IVIG over $12-24 \mathrm{~h}$ and $30 \mathrm{mg} / \mathrm{kg} / \mathrm{d}$ aspirin) or experimental group (receiving $2 \mathrm{~g} / \mathrm{kg} / \mathrm{d}$ IVIG, $30 \mathrm{mg} / \mathrm{kg} / \mathrm{d}$ aspirin, and an additional $2 \mathrm{mg} / \mathrm{kg} / \mathrm{d}$ prednisolone). Assessments of coronary arteries will be conducted at baseline, 2 weeks, 1 month, 3 months, 6 months, and 1 year of illness. The primary outcome will be the occurrence of CAL at 1 month of illness. In addition, the duration of fever, changes in laboratory data, the percentage requiring rescue therapy, changes in $Z$ scores, and occurrence of CAL at every time point of echocardiography during the study period will be compared 
between the two groups. All adverse events (AEs) will be reported. The study protocol is reported in accordance with the Standard Protocol Items: Recommendations for Clinical Interventional Trials (SPIRIT) 2013 statement (Additional File 1). A flow chart of the trial design is shown in Fig. 1. Figure 2 shows the study timeline according to the SPIRIT diagram.

The World Health Organization Trial Registration Data Set is included in the protocol to serve as a brief structured summary of the trial (Additional File 2).

\section{Eligibility criteria}

Eligible patients refer to those meeting all inclusion criteria and none of the exclusion criteria listed below:

\section{Inclusion criteria:}

1. Meeting the diagnostic criteria for KD published by the American Heart Association (AHA) in 2017 [12], including complete KD (also known as typical or classic KD) and incomplete KD (also known as atypical KD)

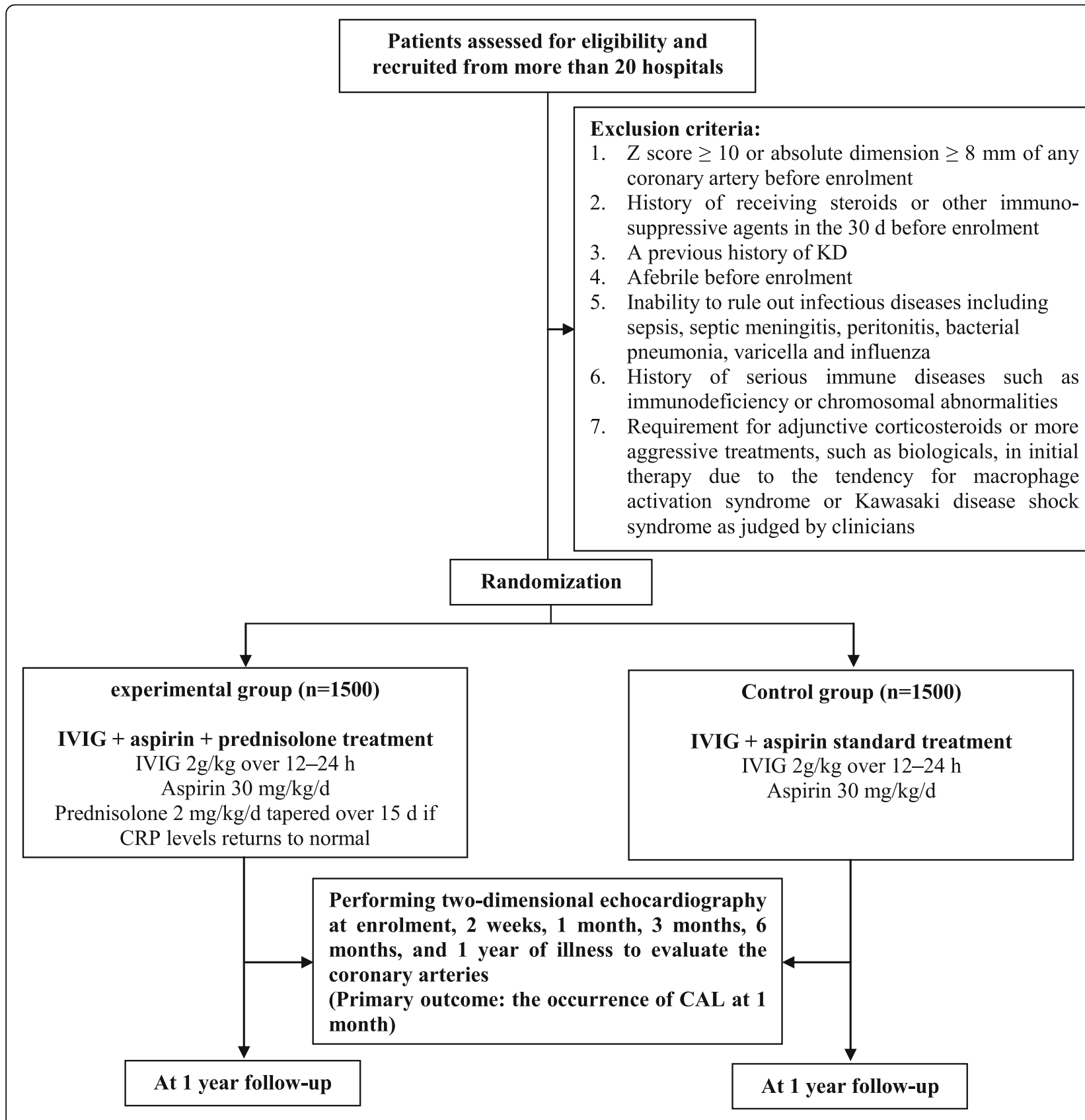

Fig. 1 Diagram of the study design. CAL, coronary artery lesion; CRP, C-reactive protein; IVIG, intravenous immunoglobulin; KD, Kawasaki disease 


\begin{tabular}{|c|c|c|c|c|c|c|c|c|c|c|c|c|c|c|c|}
\hline \multirow[b]{4}{*}{ TIMEPOINT } & \multicolumn{15}{|c|}{ STUDY PERIOD } \\
\hline & \multirow{3}{*}{\begin{tabular}{|l|} 
Enrolment \\
Before \\
IVIG
\end{tabular}} & \multirow{3}{*}{\begin{tabular}{|c|} 
Allocation \\
0
\end{tabular}} & \multicolumn{12}{|c|}{ Post-allocation } & \multirow[t]{2}{*}{ Close-out } \\
\hline & & & \multirow[b]{2}{*}{ IVIG } & \multicolumn{6}{|c|}{ From IVIG completion } & \multicolumn{5}{|c|}{ From onset of fever } & \\
\hline & & & & \begin{tabular}{|l|} 
Imme- \\
diately
\end{tabular} & \begin{tabular}{|c|}
24 \\
hours \\
\end{tabular} & \begin{tabular}{|c|}
36 \\
hours
\end{tabular} & \begin{tabular}{|c|}
60 \\
hours
\end{tabular} & $\begin{array}{c}72 \\
\text { hours }\end{array}$ & $\begin{array}{c}18 \\
\text { days }\end{array}$ & $\begin{array}{c}\text { two } \\
\text { weeks }\end{array}$ & $\begin{array}{c}\begin{array}{c}\text { one } \\
\text { month }^{\mathrm{a}}\end{array} \\
\end{array}$ & $\begin{array}{c}\text { six } \\
\text { weeks }\end{array}$ & \begin{tabular}{|c|} 
three \\
months
\end{tabular} & \begin{tabular}{c|} 
six \\
months
\end{tabular} & one year \\
\hline \multicolumn{16}{|l|}{ ENROLMENT: } \\
\hline Inclusion criteria & $x$ & & & & & & & & & & & & & & \\
\hline Exclusion criteria & $x$ & & & & & & & & & & & & & & \\
\hline Informed consent & $x$ & & & & & & & & & & & & & & \\
\hline Allocation & & $\mathrm{X}$ & & & & & & & & & & & & & \\
\hline \multicolumn{16}{|l|}{ INTERVENTIONS: } \\
\hline \multicolumn{16}{|l|}{ IVIG } \\
\hline \multicolumn{16}{|l|}{ Aspirin } \\
\hline \multicolumn{16}{|l|}{ Prednisolone $^{\mathrm{b}}$} \\
\hline \multicolumn{16}{|l|}{ Rescue therapy ${ }^{\mathrm{c}}$} \\
\hline \multicolumn{16}{|l|}{ ASSESSMENTS: } \\
\hline Baseline characteristics & $x$ & & & & & & & & & & & & & & \\
\hline Physical examination & $x$ & & & & & & & & & & & & & & \\
\hline Temperature $^{\mathrm{d}}$ & $x$ & & $\leftarrow$ & & & & $\rightarrow$ & & & & & & & & \\
\hline Laboratory examination $^{\mathrm{e}}$ & $x$ & & & & & & & $x$ & & & & & & & \\
\hline Echocardiography & $\mathrm{x}$ & & & & & & & & & $x$ & $x$ & & $x$ & $x$ & $x$ \\
\hline Other auxiliary examination ${ }^{\mathrm{f}}$ & $4 \ldots .$. & (............ & $\ldots . . .$. & ........ & $\ldots . .$. & $\ldots$ & $\ldots . .$. & $\ldots . .$. & (....... & n...... & $\cdots$ & $\ldots . . .$. & ........ & (.......... & $\cdots$ \\
\hline Adverse event & & & 4 & & & & & & & & & & & & $\longrightarrow$ \\
\hline
\end{tabular}

Fig. 2 Schedule of enrolment, intervention, and assessment. a Gray shading shows the primary endpoint. $\mathbf{b}$ It is inferred experientially that CRP levels may become normal 3 days after IVIG completion when the patient switches from intravenous methylprednisolone to oral prednisolone tapered over 15 days. c Patients will accept rescue therapy if they exhibit IVIG resistance. $\mathbf{d}$ Axillary temperature (or rectal temperature) will be measured every $6 \mathrm{~h}$ from IVIG treatment. The time point, temperature, and treatment will be recorded if fever occurs between two measurements. When IVIG starts and body temperature becomes normal it will be recorded. e Laboratory examination includes CRP, ESR, Hct, Hb, ALB, SAA, prealbumin, ALT, AST, CK-MB, sodium, NT-proBNP, IL-2, IL-4, IL-6, IL-10, TNF-a, TB, troponin, D-dimer, lipid, and lipoprotein serum levels and WBC, NEUT, and PLT counts. CRP and routine blood tests will be measured every 3 days after completion of initial IVIG infusion until normal. The remaining indicators (except for ESR), if abnormal, will also be measured every 3 days after completion of initial IVIG infusion until normal. $\mathbf{f}$ Other auxiliary examination includes electrocardiogram, chest radiography, magnetic resonance angiography, and myocardial perfusion imaging. The patient will choose whether to accept the examinations based on the physical condition during the diagnostic and therapeutic period. ALB, serum albumin; ALT, alanine aminotransferase; AST, aspartate transaminase; CK-MB, creatine kinase-muscle/brain; CRP, C-reactive protein; ESR, erythrocyte sedimentation rate; $\mathrm{Hb}$, hemoglobin; Hct, hematocrit; IL-2, interleukin-2; IL-4, interleukin-4; IL-6, interleukin-6; IL-10, interleukin-10; IVIG, intravenous immunoglobulin; NEUT, neutrophil; NT-proBNP, N-terminal pro-B-type natriuretic peptide, PLT, platelet; SAA, serum amyloid A; TB, total bilirubin; TNF-a, tumor necrosis factor-alpha; WBC, white blood cell

2. Diagnosed within 10 days of onset (including the 10th day and the 1st day of onset defined as the first day of fever)

3. Not yet treated with IVIG

4. Age $\geq 1$ month at the time of enrolment into the trial

\section{Exclusion criteria:}

1. $Z$ score $\geq 10$ or absolute dimension $\geq 8 \mathrm{~mm}$ of any coronary artery before enrolment

2. History of receiving steroids or other immunosuppressive agents 30 days before enrolment

3. A previous history of $\mathrm{KD}$

4. Afebrile (axillary temperature lower than $37.5^{\circ} \mathrm{C}$ for at least $24 \mathrm{~h}$ ) before enrolment
5. Inability to rule out infectious diseases, including sepsis, septic meningitis, peritonitis, bacterial pneumonia, varicella, and influenza

6. History of serious immune diseases, such as immunodeficiency, or chromosomal abnormalities

7. Requirement for adjunctive corticosteroids or more aggressive treatments, such as biologicals, in initial therapy due to the tendency for macrophage activation syndrome or Kawasaki disease shock syndrome as judged by clinicians

\section{Recruitment and informed consent}

The study is expected to be conducted in more than 20 hospitals in China, distributed among at least 10 provinces and municipalities in the eastern, western, northern, southern, and central parts of China. Under the 
precondition of ensuring randomization, no specific number of subjects enrolled by each participating institute will be required.

In each institute, patients diagnosed with KD will be considered as the participants in this trial. The research physician will discuss information regarding the main aspects of the trial with the parent or guardian, as well as with the patients who are determined to have the ability to understand the trial and obtain written consent from the parent or guardian if the children with KD are eligible and the parent or guardian is willing to allow them to participate in the trial. In the case of patients over the age of 10, a signed informed consent from the patient will be needed in addition to that provided by the parent or guardian.

\section{Randomization: sequence generation and allocation concealment}

According to the random seeds generated by the SAS software (version 9.4; SAS Institute, Cary, NC, USA), numbers from 1 to 6 were randomly generated by a computer for each block, and each number corresponded to one of six plans for allocating four participants of the block to two arms in a 1:1 ratio. Each allocation sequence was placed in small, opaque, and sealed envelopes numbered and marked in order from one to four and were enclosed in a larger, opaque, and sealed envelope marked with the block number. Randomization was stratified by study site. A randomization plan and concealed envelopes were prepared by an independent team of statisticians from the Clinical Trial Unit (CTU) of the Children's Hospital of Fudan University.

Recruited patients will be randomly assigned in a 1: 1 ratio to the control or experimental group through blocked randomization using the center as the stratified factor. After obtaining signed informed consent from the eligible participant, the trained physician will meet with the prespecified institutional nurse for the allocation plan for the very participant, as indicated by the corresponding envelope. Block envelopes and the four enclosed small envelopes will be required to be opened for enrolment and recruitment. The nurse will administer the allocation record form in front of the physician, and the form from each site will be monitored weekly using a data monitor from the central hospital.

\section{Blinding}

Patients and physicians will not be masked to the assignment. Pediatric cardiologists who assess CAL using echocardiography will be blinded to the assignment.

\section{Interventions}

Participants in the control group will receive IVIG $2 \mathrm{~g} /$ $\mathrm{kg}$ and oral aspirin $30 \mathrm{mg} / \mathrm{kg}$ per day (given over three doses) in the initial treatment. Participants in the experimental group will receive IVIG $2 \mathrm{~g} / \mathrm{kg}$, oral aspirin 30 $\mathrm{mg} / \mathrm{kg}$ per day (given over three doses), and prednisolone $2 \mathrm{mg} / \mathrm{kg}$ per day in the initial treatment.

IVIG will be administered over $12-24 \mathrm{~h}$ with a maximum dose of $60 \mathrm{~g}$. The dose of aspirin will be reduced to $3-5 \mathrm{mg} / \mathrm{kg}$ per day after three fever-free days and normalization of C-reactive protein (CRP) level, and its administration will continue for at least 6 weeks after the onset of KD. Patients in the experimental group will receive intravenous methylprednisolone $1.6 \mathrm{mg} / \mathrm{kg}$ per day, which is given in two doses (equal to prednisolone $2 \mathrm{mg} / \mathrm{kg}$, and the maximum dose is $60 \mathrm{mg}$ of prednisolone), then switched to oral prednisolone $2 \mathrm{mg} / \mathrm{kg}$ after three fever-free days. If CRP levels return to normal, the prednisolone dose will be tapered over 15 days in 5-day steps, from $2 \mathrm{mg} / \mathrm{kg}$ per day to $1 \mathrm{mg} / \mathrm{kg}$ per day to 0.5 $\mathrm{mg} / \mathrm{kg}$ per day. During corticosteroid administration, 0.5 $\mathrm{mg} / \mathrm{kg}$ of omeprazole will be administered daily for gastroprotection.

In both groups, patients resistant to initial IVIG therapy will receive rescue therapy, including a second dose of IVIG $(2 \mathrm{~g} / \mathrm{kg})$, a high dose of methylprednisolone (10-30 $\mathrm{mg} / \mathrm{kg}$ per day), infliximab (5 mg/kg), other immunosuppressive agents, a combination of two or more drugs, or even more aggressive treatment, such as plasmapheresis, depending on patient condition and physician experience. IVIG resistance is defined as recurrent or persistent fever (axillary temperature $\geq 37.5^{\circ} \mathrm{C}$ or rectal temperature $\left.\geq 38^{\circ} \mathrm{C}\right) 36 \mathrm{~h}$ after completion of the initial IVIG infusion.

For patients developing CALs, the investigators will follow up for at least 3 years and provide recommendations for treatment and physical activity based on the guidelines "Recommendations for clinical management of Kawasaki disease with coronary artery lesions (2020 revision)" [13] during the follow-up period.

The schedule for the study data collection is described in Fig. 2.

\section{Assessment and outcome measures}

The baseline characteristics of each patient will be collected, including sex, date of birth, height, weight, clinical manifestations, subtype of $\mathrm{KD}$, days of fever before initial IVIG, echocardiographic findings at enrolment, a series of pre-IVIG laboratory tests, and the time at which initial IVIG is administered.

Two-dimensional echocardiography will be performed to evaluate the alternation in diameters of the coronary arteries at six time points: at enrolment (before initial IVIG treatment), 2 weeks of illness $( \pm 2$ days, before 
discharge), 1 month of illness (+ 5 days), 3 months of illness ( \pm 5 days), 6 months of illness ( \pm 5 days), and 12 months of illness ( \pm 5 days). In some cases, such as for patients with severe CALs, echocardiography assessments will be more frequent and relevant results will also be recorded. The measurement for each patient's echocardiogram will include the internal diameters of the left main coronary artery (LMCA), left anterior descending artery (LAD), left circumflex coronary artery (LCX), and proximal and middle segments of the right coronary artery (RCA). Echocardiography will be performed by pediatric echocardiographers at each participating center. Video recordings will be preserved and reevaluated by two other pediatric cardiologists for confirmation. The $Z$ score of each coronary artery will be calculated based on height, weight, and measured diameter of the coronary artery [14].

\section{Primary outcome measurement}

The primary outcome will be the occurrence of CAL at 1 month of illness. According to the guidelines published by the AHA in 2017, CAL is defined as $Z \geq 2$ of any coronary arteries of the LMCA, LAD, LCX, and proximal and middle segments of the RCA and is stratified based on the maximal $Z$ score $\left(Z_{\max }\right)$ and diameters of all coronary arteries. Dilation only is defined as $Z_{\max }$ $\geq 2$ to $<2.5$; small aneurysms are defined as $Z_{\max } \geq 2.5$ to $<5$; medium aneurysms are defined as $Z_{\max } \geq 5$ to $<$ 10 and absolute dimension $<8 \mathrm{~mm}$; and giant aneurysms are defined as $Z_{\max } \geq 10$ or absolute dimension $\geq$ $8 \mathrm{~mm}$. Regression of CAL is defined as $Z<2$ of all coronary arteries (LMCA, LAD, LCX, and the proximal and middle segments of the RCA) [12].

\section{Secondary outcome measures}

1. The need for rescue therapy

2. Duration of fever (hours): from initiation of initial IVIG infusion to afebrile condition defined as axillary temperatures $<37.5^{\circ} \mathrm{C}$ or rectal temperature $<38^{\circ} \mathrm{C}$ persisting for at least $24 \mathrm{~h}$

3. Occurrence of CAL at every time point at which echocardiography was performed during the study period

4. Changes in $Z$ scores of LMCA, LAD, LCX, and the proximal and middle segments of the RCA throughout the study period (from admission to 12 months of illness)

5. Changes in serum CRP concentration $72 \mathrm{~h}$ after completion of initial IVIG infusion.

6. Frequency of AEs

\section{Safety}

All AEs that occur during the trial period, such as death, hypertension, severe infection, allergic reactions, heart failure, and thrombosis, will be recorded and followed up until their resolution or 4 weeks after the end of the trial. The physician will judge the correlation of the events with the study drugs and record the related information, including the initiation time, duration, severity, correlation, and adjustment of treatment. Serious AEs must be immediately reported to the principal investigator.

\section{Adherence}

First, face-to-face adherence reminder sessions will take place, including instructions about taking study medications, reinforcement of the need for regular follow-up, and notification of each assessment time point, which will also be recorded in the patient's discharge summary. Second, the investigators will remind the patients to accept relevant examinations at every assessment time point. Third, we have a collaboration network composed of all participating centers, which may improve adherence and reduce the loss rate of follow-up. Finally, when discharged from the hospital, each patient who developed CALs will be informed of the type and frequency of cardiology assessment during the long-term follow-up (at least 3 years), which are decided by clinical experts based on risk stratification and recommendations of guidelines published in 2020 [13]. The physician will also remind the patient of the time and items for the next visit at the end of each follow-up, in hopes that these strategies will be useful for improving adherence.

The drop out criteria include discontinuation of the allocated intervention, which will be judged by the attending pediatric cardiologists due to severe side effects.

\section{Sample size calculation}

The sample size is calculated using the SAS software (version 9.4). We assume a difference in the percentage of CAL at 1 month between the two groups of 3.3\% $(12.4 \%$ vs. 9.1\%) [10] for the control and experimental groups, respectively. A total of 1390 cases in each group would be needed with an $\alpha$ of 0.05 and a power of 0.8 . Given a potential dropout rate of $5 \%$ during follow-up, a total of 1500 patients in each group are planned to be enrolled.

\section{Statistical analysis}

The primary analysis will be an intention-to-treat analysis. Per-protocol analysis will also be performed as a supportive analysis.

The generalized estimating equation model will be used to analyze the between-group difference in repeated measurements of the primary outcome 
(occurrence of CAL at 1 month of illness) and other secondary outcomes. Subgroup analyses will be performed based on age, gender, BMI, and type of KD diagnosis (KD or incomplete $\mathrm{KD}$, defined according to the AHA diagnostic guidelines for KD, 2017 [12]) and IVIG response or not.

Descriptive statistics, such as gender, age, clinical manifestations, and subtype of $\mathrm{KD}$, will be used to determine the baseline participant demographics and general status of the patients. For non-parametric data, the MannWhitney test will be used. Variables will be checked for normal distribution and presented as mean \pm standard deviation and compared with the Student $t$ test when normally distributed. For non-normally distributed variables, the data will be expressed as median \pm interquartile range, and non-parametric tests will be used. Categorical variables will be expressed as number (\%) and analyzed using the $\chi^{2}$ tests or Fisher's exact tests when appropriate. Safety analyses will be compared with the incidence of AEs in the two groups using the $\chi^{2}$ test.

The confirmatory two-sided significance level will be set at $5 \%$ for all statistical tests and confidence intervals. SAS version 9.4 will be used for all analyses.

A detailed statistical analysis plan will be uploaded before the last enrolment to ClinicalTrials.gov (NCT04078568).

\section{Data collection, management, and monitoring}

Before the recruiting starting date, all personnel involved will be trained to ensure full understanding of the research protocol and standardized measurement of the outcomes, including the internal diameters of coronary arteries. A standard case report form (CRF) has been established to record data, such as medical history, temperature records, laboratory data, echocardiography results, and auxiliary examination results.

All data will be inputted to the electronic database created by ACCESS in a timely manner. Each institution will input data offline and will upload the dataset into a cloud disk, which will be routinely monitored by a data manager from the CTU of the Children's Hospital of Fudan University. A data monitoring committee (DMC) will be established, consisting of independent clinical experts and independent statisticians, supervising the research process according to "A proposed charter for clinical trial data monitoring committees: helping them to do their job well" [15]. Unblinded data can be accessed by members of the DMC, and the performance and safety of the trial will be reviewed weekly.

Auditing will be conducted annually, and the process will be independent from investigators.

The final closed trial dataset will be under the custody of the Children's Hospital of Fudan University. The statisticians from the CTU of the Children's Hospital of
Fudan University will have full access to the complete, anonymized final dataset. Access to the final dataset or identifiable data by others will require written application and approval by the DMC and all study investigators. The original CRFs and consent forms will also be collected and stored securely at the Children's Hospital of Fudan University for a period of 5 years after publication of the last paper or report from the study.

\section{Discussion}

Since the initial report on KD by Tomosaki Kawasaki in 1967 [16], the means of reducing the occurrence of CAL has often been an issue of focus. As an adjunctive therapy for primary treatment of $\mathrm{KD}$, corticosteroids are an affordable and relatively safe alternative for most patients, although their efficacy and safety need more evidence in non-Japanese populations.

According to the 2017 guidelines released by the AHA [12], the administration of a longer course of corticosteroids together with IVIG and aspirin is recommended for the treatment of high-risk patients with acute $\mathrm{KD}$ based on the results of the study by Kobayashi et al., but there is no consensus on the scoring systems used to identify patients at high risk for non-responsiveness to primary IVIG. Despite the extensive use of Kobayashi's score (sensitivity and specificity of $86 \%$ and $67-68 \%$, respectively) [17] and Egami's score (sensitivity and specificity of $78 \%$ and $76 \%$, respectively) [18] in screening high-risk patients in Japan, their applications are limited in other populations, including the Korean, North American, and Chinese populations, because of a lack of adequate sensitivity [19-24]. Additionally, some prediction models have been established based on the data of patients from different areas of China, such as Suzhou, Taiwan, and Beijing [21, 22, 25]. In our previous study on verifying the efficacy of the present prediction models, we found that the sensitivity and specificity were $0.272-0.799$ and $0.412-0.926$, respectively; none of them reached both sensitivity and specificity $\geq 0.75$. These results suggest that no prediction models established based on demographic characteristics, clinical manifestations, and laboratory indexes are appropriate for clinical use to assess the risk of IVIG resistance in Chinese children with KD [24]. Nonetheless, treatment with IVIG plus corticosteroids can cause a rapid reduction of cytokine levels, which are associated with damage to the vascular endothelia $[1,26,27]$. Considering the above factors, $\mathrm{KD}$ patients are targeted in this trial regardless of the predicted risk of IVIG resistance.

In the previous literature, neonatal KD is rare. Hangai et al. reviewed nationwide Japanese surveys of KD from 2001 to 2012 and identified 23 neonatal cases in total, accounting for $1 / 5500$ of patients of all ages [28]. Furthermore, there is no definite evidence for elevated risk 
of CAL in neonatal KD. Hangai et al. demonstrated that the risk of developing CALs was not significantly higher in neonates with KD than in older patients [28], results of which were consistent with another study involving KD patients $\leq 3$ months of age [29]. Conversely, another two reports on $\mathrm{KD}$ emphasized the high risk of coronary artery aneurysms in patients below 6 months of age [30, $31]$, in which the proportion of neonates was not mentioned. Thus, the effect of excluding infants $<1$ month in the final analysis may be negligible. It is believed that the use of systemic corticosteroids in newborns should be performed with great caution due to possible shortand long-term complications [32]. In consideration of safety, age was limited to 1 month and above as an inclusion criterion of this trial.

With regard to the therapeutic regimen of corticosteroids, one-off steroids and longer courses of steroids are two alternatives, and the superiority of the latter over the former has been exhibited in a meta-analysis performed by Wardle et al. [33]. In a study by Kobayashi et al., the prednisolone dose was set as $2 \mathrm{mg} / \mathrm{kg}$ per day administered over 15 days after concentrations of CRP were normalized. Consequently, patients treated with IVIG plus prednisolone defervesced more rapidly, and $2 \%$ of them had serious AEs, including elevated total cholesterol in two patients and neutropenia in one patient [9]. Based on this previous experience, the prednisolone dosage will be set as $2 \mathrm{mg} / \mathrm{kg}$ per day limited to below $60 \mathrm{mg}$, and the treatment course will be over 15 days in our trial. It is suggested that the clinical response of patients with KD may be affected by the different brands of IVIG [34], but current reports on the influence of the brands of corticosteroids and aspirin are lacking. The impact of branding on IVIG and aspirin will be balanced in the two groups in our study due to the use of blocked randomization, and there is no limit on brands of prednisolone in most previous trials [8, 9, 35-37]. The brands of the three drugs will not be restricted in this study. Additionally, we will record the brands used in every participating institution to aid in the analysis.

Although patients with KD presenting with CALs at baseline have been excluded from several trials $[9,35-$ 37], evaluation of the effect of the additional corticosteroid to conventional initial therapy on these patients has important clinical value. It has been reported that $81 \%$ of CALs associated with KD are determined on initial echocardiography, averaging 5.4 days after onset [38, 39]. Moreover, persistent fever, one of the principal clinical features, may aggravate CALs [40]. Thus, patients with KD who develop early CALs will also be included in this study. However, patients with giant aneurysms, defined as an internal luminal diameter $Z$ score $\geq 10$ or absolute dimension $\geq 8$ before the initial treatment and probably not regressing and adding to higher cardiac risk, such as ischemia, myocardial infarction, and ischemia-related death $[41,42]$, tend to accept more aggressive initial treatment, such as adjunctive infliximab. This may be beneficial to these patients by rapidly ceasing the vasculitis and preventing the deterioration of CALs [43, 44], compared to corticosteroids. In view of the balance between the clinical application and interests of patients, the highest $Z$ score and absolute dimension of any coronary artery is required to be less than 10 and $8 \mathrm{~mm}$ before enrolment, respectively. However, the month after the onset of KD is crucial. Coronary dilatation subsiding within 1 month is considered as transient dilatation, and coronary artery sequelae are defined as coronary aneurysms persisting for longer in Japanese guidelines [39]. As indicated by a retrospective study, coronary severity 1 month after KD onset is closely related to late coronary outcomes [45]. In addition, the high persistence probability of medium and giant aneurysms that add to cardiovascular risk at 1 year has been described; however, approximately two-thirds of the acute myocardial infarction occurs within the first year of KD onset [45]. This suggests that 1 month and 1 year of illness may be key points for observing the long-term outcomes of KD. Therefore, we set the primary and final endpoint as 1 month and 1 year of illness, respectively, to assess not only whether corticosteroids could decrease cardiovascular sequelae, but also the therapeutic effect on the CALs identified before treatment.

\section{Trial status}

Recruitment started on January 13, 2020, is ongoing at more than 20 hospitals in China and is expected to be completed in December 2021 or until a total of 3000 participants have been recruited. According to the study protocol, each patient will be followed up for 1 year. The current protocol is version 03 (January 6, 2020). At the time of manuscript submission, 1391 participants have been recruited in total, and all participants have been followed up.

\section{Abbreviations}

AE: Adverse event; AHA: American Heart Association; CAL: Coronary artery lesion; CRF: Case report form; CRP: C-reactive protein; CTU: Clinical Trial Unit; DMC: Data monitoring committee; IVIG: Intravenous immunoglobulin; KD: Kawasaki disease; LAD: Left anterior descending artery; LCX: Left circumflex coronary artery; LMCA: Left main coronary artery; RCA: Right coronary artery; SPIRIT: Standard Protocol Items: Recommendations for Clinical Interventional Trials

\section{Supplementary Information}

The online version contains supplementary material available at https://doi. org/10.1186/s13063-021-05807-3

Additional file 1. SPIRIT checklist. A completed SPIRIT 2013 checklist. Additional file 2. World Health Organization Trial Registration Data Set (Version 1.3.1). 


\section{Acknowledgements}

We would like to thank Conway Niu (Royal Aberdeen Children's Hospital, Aberdeen, Scotland) and Editage for their help in polishing the manuscript. The study group investigators

Principal investigator and research physician: Guo-Ying Huang, Fang Liu, Xiaohui Liu, Xian-Mei Huang, Si-Lin Pan, Zi-Pu Li, Yan Li, Zhen-Yu Xiong, Ying-Jun Feng, Ling Sun, Cui-Fen Zhao, Hua Zhu, Yan-Ling Liao, Shi-Wei Yang, Ping Huang, Jing-Wei Sun, Ming-Guo Xu, Xian-Yi Yu, Yi-Ling Liu, Qian Peng, XiaoYan Liu, Xiang-Yu Dong, Hua Peng, Jin-Hua Piao, Jin-Dou An, Xin-Jiang An, Rong-Zhou Wu, Min Huang, Li-Jun Qin, Juan-Li Wang, Lan He, Yi-Xiang Lin, Yin-Yin Cao, Xiao-Li Li, Gang Luo, Zhi-Xian Ji, Yu Liang, Yuan-Yuan Zhu, FangJie Wang, Ye Chen, Hai-Zhao Zhao, Yan-Yan Liang, Xiao-Li Liu, Jie Yin, Wei Li, Ying Xie, Wei-Qun Tan, Yun-Ming Xu, Fei-Fei Si, Yi-Zhou Wen, Xiao-Ping Hu, Lei Zhang, Li Min, Qiu-Peng Wang, Yong-Hua Yuan, Lian-Hua Jin, Song Feng, Xiao-Hui Cong, Han Zhang, Xiao-Bi Huang

Steering committee: Guo-Ying Huang, Fang Liu, Wei-Li Yan

Trial management committee: Guo-Ying Huang, Fang Liu, Wei-Li Yan, Lan He, Yin Wang, Yi-Xiang Lin, Yin-Yin Cao, Si-Yuan Lin, Li-Ping Xie, Xiao-Hui Liu, Xiao-Li Li, Gang Luo, Zhi-Xian Ji, Yu Liang, Yuan-Yuan Zhu, Fang-Jie Wang, Ye Chen, Hai-Zhao Zhao, Yan-Yan Liang, Xiao-Li Liu, Jie Yin, Wei Li, Jing-Wei Sun, Ying Xie, Wei-Qun Tan, Yun-Ming Xu, Fei-Fei Si, Yi-Zhou Wen, Xiao-Ping Hu, Lei Zhang, Li Min, Qiu-Peng Wang, Yong-Hua Yuan, Lian-Hua Jin, Song Feng, Xiao-Bi Huang, Han Zhang, Xiao-Hui Cong

Data manager: Yin Wang, Lan He, Ya-Lan Dou.

\section{Authors' contributions}

$\mathrm{G}-\mathrm{YH}$ and $\mathrm{FL}$ were the chief investigators; they conceived the study and led the proposal and protocol development. The protocol was written by $L H, L-$ $P X, Y W, Y-Y C, Y-X L$, and S-YL and was critically reviewed by G-YH, FL, and $W-$ LY. W-LY was the lead trial methodologist and statistician. LH, YW, and S-YL drafted the manuscript. YW and W-LY drafted the SAP. All authors have read and approved the final manuscript.

\section{Funding}

The present study was funded by the Innovation Unit Project of the Chinese Academy of Medical Sciences (2018RU002). The funder does not influence the design of the study; collection, analysis, and interpretation of the data; or writing of the manuscript. No financial or personal relationships with individuals or organizations have inappropriately influenced our work.

\section{Availability of data and materials}

The trial results will be published in a peer-reviewed scientific paper and through oral presentations at conferences. The datasets analyzed during the current study are available from the corresponding author upon reasonable request.

\section{Declarations}

\section{Ethics approval and consent to participate}

The study was approved by the Institutional Review Board of the Children's Hospital of Fudan University (number: 2018-142-01; amendment number: 2018-142-03). The date of preliminary ethics approval was April 3, 2018, and the date of final ethics approval was January 7,2020 . The final date and version of the study protocol is 03 and January 6, 2020. This trial was declared and registered at ClinicalTrials.gov, NCT04078568. Written informed consent to participate was obtained from all participants.

Protocol modifications (e.g., changes to eligibility criteria, outcomes, analyses) will be reported to the relevant parties: the investigators of participating institutions, ClinicalTrials.gov, and the local ethics committee.

\section{Consent for publication}

Not applicable.

\section{Competing interests}

The authors declare that they have no competing interests.
Received: 21 December 2020 Accepted: 8 November 2021

Published online: 11 December 2021

\section{References}

1. Burns JC, Glode MP. Kawasaki syndrome. Lancet. 2004;364(9433):533-44. https://doi.org/10.1016/S0140-6736(04)16814-1.

2. Makino N, Nakamura Y, Yashiro M, Kosami K, Matsubara Y, Ae R, et al. Nationwide epidemiologic survey of Kawasaki disease in Japan, 2015-2016. Pediatr Int. 2019;61(4):397-403. https://doi.org/10.1111/ped.13809.

3. Xie LP, Yan WL, Huang M, Huang MR, Chen S, Huang GY, et al. Epidemiologic features of Kawasaki ddisease in Shanghai from 2013 through 2017. J Epidemiol. 2019;30(10):429-35. https://doi.org/10.2188/jea. JE20190065.

4. Gordon JB, Kahn AM, Burns JC. When children with Kawasaki disease grow up: myocardial and vascular complications in adulthood. J Am Coll Cardiol. 2009;54(21):1911-20. https://doi.org/10.1016/j.jacc.2009.04.102.

5. Mori M, Miyamae T, Imagawa T, Katakura S, Kimura K, Yokota S. Metaanalysis of the results of intravenous gamma globulin treatment of coronary artery lesions in Kawasaki disease. Mod Rheumatol. 2004;14(5):361-6. https:// doi.org/10.3109/s10165-004-0324-3.

6. Terai M, Shulman ST. Prevalence of coronary artery abnormalities in Kawasaki disease is highly dependent on gamma globulin dose but independent of salicylate dose. J Pediatr. 1997;131(6):888-93. https://doi. org/10.1016/S0022-3476(97)70038-6.

7. Wooditch AC, Aronoff SC. Effect of initial corticosteroid therapy on coronary artery aneurysm formation in Kawasaki disease: a meta-analysis of 862 children. Pediatrics. 2005;116(4):989-95. https://doi.org/10.1542/peds.20050504.

8. Newburger JW, Sleeper LA, McCrindle BW, Minich LL, Gersony W, Vetter VL, et al. Randomized trial of pulsed corticosteroid therapy for primary treatment of Kawasaki disease. N Engl J Med. 2007;356(7):663-75. https:// doi.org/10.1056/NEJMoa061235.

9. Kobayashi T, Saji T, Otani T, Takeuchi K, Nakamura T, Arakawa H, et al. Efficacy of immunoglobulin plus prednisolone for prevention of coronary artery abnormalities in severe Kawasaki disease (RAISE study): a randomised, open-label, blinded-endpoints trial. Lancet. 2012;379(9826):1613-20. https:// doi.org/10.1016/S0140-6736(11)61930-2.

10. Zhu BH, Lv HT, Sun L, Zhang JM, Cao L, Jia HL, et al. A meta-analysis on the effect of corticosteroid therapy in Kawasaki disease. Eur J Pediatr. 2012; 171(3):571-8. https://doi.org/10.1007/s00431-011-1585-4.

11. Chen S, Dong Y, Kiuchi MG, Wang J, Li R, Ling Z, et al. Coronary artery complication in Kawasaki disease and the importance of early intervention: a systematic review and meta-analysis. JAMA Pediatr. 2016;170(12):1156-63. https://doi.org/10.1001/jamapediatrics.2016.2055.

12. McCrindle BW, Rowley AH, Newburger JW, Burns JC, Bolger AF, Gewitz M, et al. Diagnosis, treatment, and long-term management of Kawasaki disease: a scientific statement for health professionals from the American Heart Association. Circulation. 2017;135(17):e927-e99. https://doi.org/10.1161/CIR. 0000000000000484.

13. The Subspecialty Group of Cardiology, the Society of Pediatrics, Chinese Medical Association, Editorial Board, Chinese Journal of Pediatrics. Recommendations for clinical management of Kawasaki disease with coronary artery lesions (2020 revision). Chin J Pediatr. 2020;58(9):718-24 (in Chinese).

14. Dallaire F, Dahdah N. New equations and a critical appraisal of coronary artery Z scores in healthy children. J Am Soc Echocardiogr. 2011;24(1):60-74. https://doi.org/10.1016/j.echo.2010.10.004.

15. DAMOCLES Study Group, NHS Health Technology Assessment Programme. A proposed charter for clinical trial data monitoring committees: helping them to do their job well. Lancet. 2005;365(9460):711-22. https://doi.org/1 0.1016/S0140-6736(05)17965-3.

16. Kawasaki T. Acute febrile mucocutaneous syndrome with lymphoid involvement with specific desquamation of the fingers and toes in children. Arerugi. 1967;16(3):178-222.

17. Kobayashi T, Inoue Y, Takeuchi K, Okada Y, Tamura K, Tomomasa T, et al. Prediction of intravenous immunoglobulin unresponsiveness in patients with Kawasaki disease. Circulation. 2006;113(22):2606-12. https://doi.org/1 0.1161/CIRCULATIONAHA.105.592865.

18. Egami K, Muta $H$, Ishii M, Suda K, Sugahara $Y$, lemura M, et al. Prediction of resistance to intravenous immunoglobulin treatment in patients with Kawasaki disease. J Pediatr. 2006;149(2):237-40. https://doi.org/10.1016/j. jpeds.2006.03.050. 
19. Shin J, Lee H, Eun L. Verification of Current Risk Scores for Kawasaki Disease in Korean Children. J Korean Med Sci. 2017;32(12):1991-6. https://doi.org/1 0.3346/jkms.2017.32.12.1991.

20. Sleeper LA, Minich LL, McCrindle BM, Li JS, Mason W, Colan SD, et al. Evaluation of Kawasaki disease risk-scoring systems for intravenous immunoglobulin resistance. J Pediatr. 2011;158(5):831-5.e3.

21. Tang Y, Yan W, Sun L, Huang J, Qian W, Ding Y, et al. Prediction of intravenous immunoglobulin resistance in Kawasaki disease in an East China population. Clin Rheumatol. 2016;35(11):2771-6. https://doi.org/10.1007/s1 0067-016-3370-2.

22. Lin MT, Chang CH, Sun LC, Liu HM, Chang HW, Chen CA, et al. Risk factors and derived formosa score for intravenous immunoglobulin unresponsiveness in Taiwanese children with Kawasaki disease. J Formos Med Assoc. 2016;115(5):350-5. https://doi.org/10.1016/j.jfma.2015.03.012.

23. Lan $X$, Jing $Z$, Lunyu $Y$, Ling $Q$, Ying $Y$, Xiaochun $Y$. Predictive analysis of intravenous immunoglobulin unresponsive Kawasaki disease. J Clin Pediatr. 2018:36(10):765-71 (in Chinese)

24. Li-ping $X$, Juan G, Yang F, Lan H, Chen C, Wei-li Y, et al. Questioning the establishment of clinical prediction model for intravenous immunoglobulin resistance in children with Kawasaki disease. Chin J Evid Based Pediatr. 2019; 14(3):169-75 (in Chinese).

25. Fu PP, Du ZD, Pan YS. Novel predictors of intravenous immunoglobulin resistance in Chinese children with Kawasaki disease. Pediatr Infect Dis J. 2013;32(8):e319-23. https://doi.org/10.1097/INF.0b013e31828e887f.

26. Okada Y, Shinohara M, Kobayashi T, Inoue Y, Tomomasa T, Kobayashi T, et al. Effect of corticosteroids in addition to intravenous gamma globulin therapy on serum cytokine levels in the acute phase of Kawasaki disease in children. J Pediatr. 2003;143(3):363-7. https://doi.org/10.1067/S0022-3476(03 )00387-1.

27. Leung DY, Cotran RS, Kurt-Jones E, Burns JC, Newburger JW, Pober JS. Endothelial cell activation and high interleukin-1 secretion in the pathogenesis of acute Kawasaki disease. Lancet. 1989;2(8675):1298-302. https://doi.org/10.1016/s0140-6736(89)91910-7.

28. Hangai M, Kubota Y, Kagawa J, Yashiro M, Uehara R, Nakamura Y, et al. Neonatal Kawasaki disease: case report and data from nationwide survey in Japan. Eur J Pediatr. 2014;173(11):1533-6. https://doi.org/10.1007/s00431014-2347-x.

29. Lee EJ, Park YW, Hong YM, Lee JS, Han JW. Epidemiology of Kawasaki disease in infants 3 months of age and younger. Korean J Pediatr. 2012; 55(6):202-5. https://doi.org/10.3345/kjp.2012.55.6.202.

30. Salgado AP, Ashouri N, Berry EK, Sun X, Jain S, Burns JC, et al. High risk of coronary artery aneurysms in infants younger than 6 months of age with Kawasaki disease. J Pediatr. 2017;185:112-6.e1.

31. Singh S, Agarwal S, Bhattad S, Gupta A, Suri D, Rawat A, et al. Kawasaki disease in infants below 6 months: a clinical conundrum. Int J Rheum Dis. 2016;19(9):924-8. https://doi.org/10.1111/1756-185X.12854.

32. Committee on Fetus and Newborn. Postnatal corticosteroids to treat or prevent chronic lung disease in preterm infants. Pediatrics. 2002;109(2):3308. https://doi.org/10.1542/peds.109.2.330.

33. Wardle AJ, Connolly GM, Seager MJ, Tulloh RM. Corticosteroids for the treatment of Kawasaki disease in children. Cochrane Database Syst Rev. 2017;1(1):CD011188. https://doi.org/10.1002/14651858.CD011188.pub2.

34. Tsai MH, Huang YC, Yen MH, Li CC, Chiu CH, Lin PY, et al. Clinical responses of patients with Kawasaki disease to different brands of intravenous immunoglobulin. J Pediatr. 2006;148(1):38-43. https://doi.org/10.1016/j. jpeds.2005.08.024.

35. Ogata S, Ogihara Y, Honda T, Kon S, Akiyama K, Ishii M. Corticosteroid pulse combination therapy for refractory Kawasaki disease: a randomized trial. Pediatrics. 2012;129(1):E17-23. https://doi.org/10.1542/peds.2011-0148.

36. Inoue Y, Okada Y, Shinohara M, Kobayashi T, Kobayashi T, Tomomasa T, et al. A multicenter prospective randomized trial of corticosteroids in primary therapy for Kawasaki disease: clinical course and coronary artery outcome. J Pediatr. 2006;149(3):336-41. https://doi.org/10.1016/j.jpeds.2006. 05.025 .

37. Sundel RP, Baker AL, Fulton DR, Newburger JW. Corticosteroids in the initial treatment of Kawasaki disease: report of a randomized trial. J Pediatr. 2003; 142(6):611-6. https://doi.org/10.1067/mpd.2003.191.

38. Dominguez SR, Anderson MS, El-Adawy M, Glode MP. Preventing coronary artery abnormalities: a need for earlier diagnosis and treatment of Kawasaki disease. Pediatr Infect Dis J. 2012;31(12):1217-20. https://doi.org/10.1097/INF. Ob013e318266bcf9.
39. JCS Joint Working Group. Guidelines for diagnosis and management of cardiovascular sequelae in Kawasaki disease (JCS 2008)--digest version. Circ J. 2010;74(9):1989-2020. https://doi.org/10.1253/circj.CJ-10-74-0903.

40. Muniz JC, Dummer K, Gauvreau K, Colan SD, Fulton DR, Newburger JW. Coronary artery dimensions in febrile children without Kawasaki disease. Circ Cardiovasc Imaging. 2013;6(2):239-44. https://doi.org/10.1161/CIRCIMA GING.112.000159.

41. Kato H, Sugimura T, Akagi T, Sato N, Hashino K, Maeno Y, et al. Long-term consequences of Kawasaki disease. A 10- to 21-year follow-up study of 594 patients. Circulation. 1996;94(6):1379-85. https://doi.org/10.1161/01.CIR.94. 6.1379 .

42. Tsuda E, Hamaoka K, Suzuki H, Sakazaki H, Murakami Y, Nakagawa M, et al. A survey of the 3-decade outcome for patients with giant aneurysms caused by Kawasaki disease. Am Heart J. 2014;167(2):249-58. https://doi.org/10.101 6/j.ahj.2013.10.025.

43. Tremoulet AH, Jain S, Jaggi $P$, Jimenez-Fernandez S, Pancheri JM, Sun $X$, et al. Infliximab for intensification of primary therapy for Kawasaki disease: a phase 3 randomised, double-blind, placebo-controlled trial. Lancet. 2014; 383(9930):1731-8. https://doi.org/10.1016/S0140-6736(13)62298-9.

44. Jone PN, Anderson MS, Mulvahill MJ, Heizer H, Glode MP, Dominguez SR. Infliximab plus intravenous immunoglobulin (IVIG) versus IVIG alone as initial therapy in children with Kawasaki disease presenting with coronary artery lesions: is dual therapy more effective. Pediatr Infect Dis J. 2018; 37(10):976-80. https://doi.org/10.1097/INF.0000000000001951.

45. Lin MT, Sun LC, Wu ET, Wang JK, Lue HC, Wu MH. Acute and late coronary outcomes in 1073 patients with Kawasaki disease with and without intravenous gamma-immunoglobulin therapy. Arch Dis Child. 2015;100(6): 542-7. https://doi.org/10.1136/archdischild-2014-306427.

\section{Publisher's Note}

Springer Nature remains neutral with regard to jurisdictional claims in published maps and institutional affiliations.

Ready to submit your research? Choose BMC and benefit from:

- fast, convenient online submission

- thorough peer review by experienced researchers in your field

- rapid publication on acceptance

- support for research data, including large and complex data types

- gold Open Access which fosters wider collaboration and increased citations

- maximum visibility for your research: over $100 \mathrm{M}$ website views per year

At BMC, research is always in progress.

Learn more biomedcentral.com/submissions 